\title{
Modulation of carcinogenicity and mutagenicity by herbal medicinal plant Bacopa monnieri extract in swiss albino mice
}

\author{
R.C.Agrawal*, Vishnoi Shilki, Neha Agrawal \\ Department of Research, Priyamvada Birla Cancer Research Institute, Satna, Madhya Pradesh, India
}

\begin{abstract}
In the present investigation the topical application of single dose of DMBA followed by croton oil induced the skin papillomas in all the animals by the $15^{\text {th }}$ week. Cumulative number of Papillomas and tumor yields were recorded as 28 , and 4.6 respectively. Whereas the animals which received Bacopa monnieri extract additionally at the dose of $200 \mathrm{mg} / \mathrm{kg}$ body weight, the cumulative number of Papillomas, tumor yields were recorded as 18 and 3.0 respectively and these were observed to be significantly lower than DMBA+Croton oil group. In another experiment, B16F10 melanoma propagated by subcutaneous injection of $10^{6}$ melanoma cells on the dorsal skin of C57BL mice. The tumour growth and tumor volume of cyclophosphamide treated by subcutaneous route at single dose of $300 \mathrm{mg} / \mathrm{kg}$ body weight, $30 \mathrm{~min}$. after tumor cell inoculation the tumors size was significantly reduced and inhibited the tumors growth by $46.74 \%$. Whereas when cyclophosphamide was given at single dose and Bacopa monniere extract was given for 21 days by oral route, the tumor size was further reduced and tumor growth was inhibited by $\mathbf{5 0 . 7 1 \%}$. However Bacopa monniera ext. alone treatment $(200 \mathrm{mg} / \mathrm{kg}$ oral 21 days) the tumour volume was also reduced and tumor growth was inhibited by $27.27 \%$. It seems that Bacopa monnieri extract in combination with cyclophosphamide has significantly prevented the tumour volume as compared to control.

In antimutagenicity studies Bacopa monnieri administered i.p. at the dose dependent manner have inhibited micronucleus formation and chromosomal aberrations induced by known mutagen in Bone marrow cells of Swiss albino mice. It is therefore concluded that Bacopa monnieri extract has anticarcinogenic and antimutagenic activity in the above test systems.
\end{abstract}

Keywords: Bacopa monnieri, Skin Papilloma, Melanoma, Micronucleus, Chromosomal aberrations, Chemoprevention.

Accepted on 21 February, 2017

\section{Introduction}

To cure human disease, medicinal plants has been a major source of therapeutic agents since ancient times. The revival of interest in natural drugs started in last decade mainly because of the wide spread belief that green medicine is healthier than synthetic products. Nowadays, there is manifold increase in medicinal plant based industries due to the increase in the interest of use of medicinal plants throughout the world which are growing at a rate of $7-15 \%$ annually. Despite the major advances in the modern medicine, the development of new drugs from natural products is still considered important. This seems to be even more relevant for the developing countries, where the cost to develop a drug is prohibitive. Since 1980, the World Health Organization has been encouraging countries to identify and exploit traditional medicine and phytotherapy. The main Indian Traditional System of Medicine namely Ayurveda and Siddha are primarily plant based system. The evaluation of new drugs especially phytochemically obtained materials has again opened a vast area for research and development. As per WHO, about $80 \%$ of the population in the world relays on the traditional medicine for the treatment of various diseases. Every year, millions of people are diagnosed with Cancer, leading to death in a majority of the cases. According to the American Cancer Society, deaths arising from cancer constitute
$2-3 \%$ of the annual deaths recorded worldwide. Cancer rates are increasing every year; breast cancer being the most common form of cancer in women worldwide and the second most common cancer amongst South African women. Current statistics indicates that across all ethnic groups, one in every 31 women in this country is likely to develop breast cancer. Many traditional healers and herbalists in the Eastern Cape of S.A have been treating cancer patients for many years using various medicinal plant species.

Bacopa monnieri is called "Brahmi," in Hindi which has been used as a Brain tonic in traditional system of medicine. It also provides relief in patients with anxiety or epileptic disorders. The plant has also been used as a cardiac tonic, digestive aid and improves respiratory function. Hepatoprotective, antioxidant, anti-inflammatory and immunostimulant actions. Free radical scavenging property was well reported. The effect of standardized hydroalcoholic lyophilized extract of Bacopa monnieri in isoproterenol (ISP)-induced myocardial necrosis was studied [1]. Free radical scavenging capacity and protective effect of Bacopa monnieri L. on DNA damages [2] and anti-inflammatory effects in rodents and on antiHelicobacter pylori activity and accumulation of prostaglandins [3] have been reported [4]. In one incomplete in vitro study on the anticancer activity of Bacopa monnieri have been observed [5]. Since there are insufficient studies are available regarding 
Citation: Agrawal RC, Shilki V, Agrawal N. Modulation of carcinogenicity and mutagenicity by herbal medicinal plant Bacopa monnieri extract in swiss albino mice. J Mol Oncol Res. 2017;1(1):1-3.

the anticancer activity of Bacopa monnieri extract, we have therefore undertaken to study in the experimental animals.

\section{Materials and Methods}

\begin{abstract}
Animals
The Swiss albino and C57 Bl hybrid mice of 6-8 weeks old and 20-25 g body weight were obtained from the animal colony of PBCRI (Priyamvada Birla Cancer Research Institute, Satna). They were kept in controlled temperature and 12: $12 \mathrm{~h}$ light and dark cycle as per the protocol of CPCSEA ( Committee for the purpose of Control and supervision of experiments ) on Animals and were given synthetic diet and water ad libitum. The experiments were approved by the institutional Animal ethics committee before conduction of the experiments.
\end{abstract}

Chemicals: Dimethylbenz (a) anthracene, (DMBA) croton oil, cyclophosphamide was purchased from Sigma chemicals co. USA and other chemicals were reagent grade and procured from Ranbaxy and other companies.

\section{Preparation of Bacopa monnieri extract}

Bacopa monnieri (BM) plants were collected from the local garden in September 2008 and were identified by the competent botanist of local laboratory at Nagpur, India. Plants were air dried in shade without direct exposure to sun rays and it was powdered. The powder was mixed in $50 \%$ methanol and kept in separating funnel for 6 hours. The supernatant was collected and this process was repeated until clear solution of supernatant was obtained. All supernatant was pooled together and dried to the powder at $40^{\circ} \mathrm{C}$ water bath. The powder was dissolved in double distilled water before the each treatment at required concentrations. The Extracted material was used for preliminary photochemical screening, saponine glycosides content, alkaloids, and flavonoid content were determined.

\section{Experimental design}

Papilloma model: The animals were divided into five different groups as follows:

Group I: (initiator control) DMBA alone $100 \mu \mathrm{g}$ DMBA was dissolved in $100 \mu \mathrm{l}$ acetone and was given single application.

Group II: (promoter control) Croton oil alone 1\% Croton oil was applied on skin three times in week, up to 16 weeks.

Group III: (carcinogen control) DMBA + Croton Oil $100 \mu \mathrm{g}$ DMBA was dissolved in $100 \mu \mathrm{l}$ acetone and single application was given and croton oil was applied on skin three times in week two week later after DMBA applications for 16 weeks.

Group IV: DMBA + Croton Oil + Bacopa monnieri extract 100 $\mu \mathrm{g}$ DMBA was dissolved in $100 \mu \mathrm{l}$ acetone and single application was given and croton oil was applied on skin three times in week two week later after DMBA applications. Bacopa monnieri extract was given an hour before each application of croton oil.

Group V: (vehicle control) Acetone alone $100 \mu$ l acetone topical applied thrice a week until end of experiment.
Melanoma model: Melanoma assay was done by method standardized by Agrawal et al. [6]. The control and treated groups were maintained.

Control Group: The control group consists of four mice. The melanoma cell lines (B6 F10) were injected subcutaniousely (s.c.) in all four mice.

Test Group: This group was divided into two sub groups. First group is given Bacopa monneri extract with known anti-cancer drug Cyclophosphamide and second group was given Bacopa monnieri extract alone. Each group consisted of four animals. The melanoma cell line was injected by s.c. route in tumour bearing mice were orally given 50\% methonolic extract of Bacopa monneri at the dose of $200 \mathrm{mg} / \mathrm{Kg}$ body weight as standardized by us in earlier experiments [7].

\section{Cytotoxic activity}

It was done by the bone marrow chromosomal aberration and micronucleus assay as prescribed by the method of Schimidt et al. [8] and standardized by Agrawal et al. [6,9]. Bacopa monnieri leaves extract at different dose levels were injected $24 \mathrm{~h}$ before the treatment of cyclophosphamide and bone marrow was aspirated as per method described by Schimidt et al. [8] and standardized by Us Agrawal et al. [6,9].

\section{Results}

Topical application of single dose of DMBA followed by croton oil resulted the development of skin Papillomas in all animals, which started appearing from $5^{\text {th }}$ week onward. The incidence of tumor reached $100 \%$ by the 15 th week. Cumulative number of Papillomas, tumor yields and tumor multiplicity were recorded as $28,4.6$ and 4.6 respectively. The animals which received Bacopa monnieri extract additionally at the dose of $200 \mathrm{mg} / \mathrm{kg}$ body weight, all six animals developed skin Papillomas However Cumulative number of Papillomas, tumor yields were recorded as 18 and 3.0 respectively and these were observed significantly lower than DMBA+Croton oil group. Bacopa monniera extract has prevented the development of cumulative number of skin papillomas as compared to DMBA + Croton oil. However Bacopa monnieri have shown anti-promoting activity as compared to carcinogen control group.

In another experiment, B16F10 melanoma propagated by subcutaneous injection of $10^{6}$ cells on the dorsal skin of C57BL mice. The tumour growth and tumor volume of control group which was measured $1847.06 \mathrm{~mm}^{3}$. When Cyclophosphamide was injected s.c. at a single dose of 300 $\mathrm{mg} / \mathrm{kg}, 30 \mathrm{~min}$. after tumor cell inoculation. Tumors size was measured $983.57 \mathrm{~mm}^{3}$ and tumors growth inhibition was found to be $46.74 \%$. In another group, Cyclophosphamide was given at single dose and Bacopa monnieri extract by oral route was given for 21 days. Tumor size of BM + Cyclo group was measured $910.39 \pm 37.65 \mathrm{~mm}^{3}$ and tumor growth inhibition was found to be $50.71 \%$. In Bacopa monnieri ext. alone treatment (200 mg/kg oral 21 days) after tumor cell inoculation tumors size was measured $1343.35 \mathrm{~mm}^{3}$ and tumor growth inhibition was found to be $27.27 \%$. It seems that Bacopa 
monneri extract in combination with cyclophosphamide have significantly prevented the tumour volume as compared to control (Figures 1 and 2).

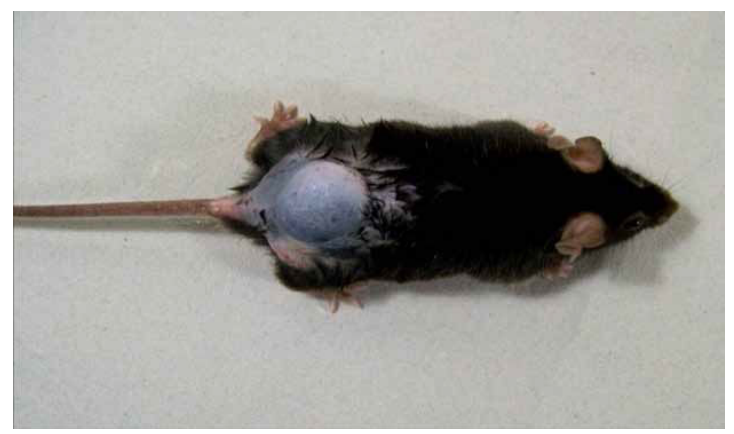

Figure 1: B16F10 melanoma tumor in Control untreated group.

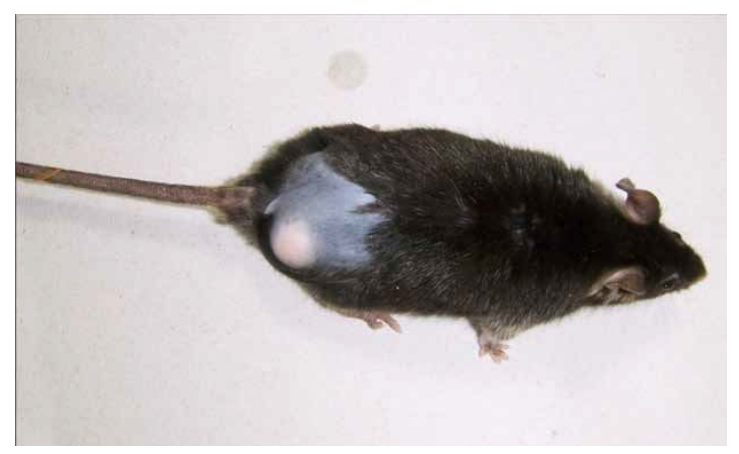

Figure 2: B16F10 reduced melanoma tumor in treated group.

In antimutagenicity studies Bacopa monnieri administered i.p. at the dose of 50,100 and $200 \mathrm{mg} / \mathrm{kg}$ body weight was found to inhibit the micronuclei formation induced by cyclophosphamide given i.p. at the dose of $50 \mathrm{mg} / \mathrm{kg}$ body weight. A dose dependent response was remarkable and statistically significant. It is noteworthy that different doses of Bacopa monnieri used in the present experiment were not cytotoxic which indicate no changes of PCE/NCE ratio (Polychromatic erythrocyte/Normochromatic erythrocyte). In case of chromosomal aberration test, there was a significant protection in chromosomal aberrations in all group of Cyclophosphamide and Bacopa monnieri extract with the dose of $50,100,200 \mathrm{mg} / \mathrm{kg}$ body weight and \% protection was found to be $18.98,26.67$ and 46.36 respectively as compared to positive control. Thus, tested Bacopa monnieri extract seems to have a preventive potential against cyclophosphamide induced chromosomal aberrations in Swiss mouse bone marrow cells.

\section{Discussion}

In present study, a single i.p. administration of Bacopa monnieri ext. resulted in dose dependent inhibition of micronuclei formation and prevented chromosomal aberrations in mouse bone marrow cells induced by cyclophosphamide. Prevention of micronucleus and chromosomal aberrations by Bacopa monnieri was helpful for prevention for cancer and mutagenecity. In present investigation for solid tumor (skin papilloma) and melanoma from above dose was selected as $200 \mathrm{mg} / \mathrm{kg}$. These findings establish their normal tissue protective efficacy. While using a drug for normal tissue protection in cancer therapy, it is highly desirable that the drug should give significant protection to normal tissues with no or minimal protection to tumors. The exact mechanism of tumour protection by Bacopa extract is still not known However it is believed that its effect may be any of the mechanism such as due to cytotoxic activity by Sarcoma -180 cell line, inhibition of DNA replication in Cancer cells, preventing the active carcinogen from their active precursors or by augmenting detoxification process, preventing promotional events in the mouse skin through free radical scavenging mechanism. The Free radical scavenging activity using Antioxidant activity and elevation of Glutathione levels has been also investigated [10]. From the above study it was concluded that $50 \%$ methanolic extract of Bacopa monniera, showed anticarcinogenic and antimutagenic activity.

\section{References}

1. Nandeve M, Ojha S. Cardioprotective effect of Bacopa monnieri against isoproterenol induced myocardial necrosis in rats. A Bibliography of Indian Medicine 2007;3:385-92.

2. Russo A, Izzo A, Borrelli F. Free radical scavenging capacity and protective effect of Bacopa monniera L. on DNA damage. Phytother Res. 2003;17:870-75.

3. Goel RK, Sairam K, Babu MD. In vitro evaluation of Bacopa monniera on anti- Helicobacter pylori activity and accumulation of prostaglandins. Phytomedicine. 2003; 10: 523-7.

4. Jain P, Khanna NK, Trehan TN. Anti-inflammatory effects of an Ayurvedic preparation, Brahmi Rasayan, in rodents. Indian J Exp Biol. 1994;32:633-6.

5. Elangovan V, Govindasamy S, Ramamoorthy N, et al. In vitro studies on the anticancer activity of Bacopa monnieri. Fitoterapia. 1995;66:211-5.

6. Agrawal RC, Kumar S. Prevention of chromosomal aberrations in mouse bone marrow by Indole-3-carbinol. Toxicol Lett. 1999;106:137-41.

7. Agrawal RC, Jain R, Wasim Raja, et al. Anticarcinogenic effect of Solanum lycopersicum fruit extract on Swiss Albino and C57 BL mice. Asian Pacific Journal of Cancer prevention. 2009;10:379-82.

8. Schmid W. The micronucleus test. Mutat Res. 1975;31:9-15.

9. Agrawal RC, Kumar S. Prevention of cyclophosphamide induced Micronucleus formation in mouse bone marrow by Indole-3-carbinol. Food Chem Toxicol. 1998;36:975-7.

10. Shilki V. Ph.D. Thesis, Barkatullah University, Bhopal. (2013).

\section{*Correspondence to}

\section{R.C. Agrawal}

Department of Research, Priyamvada Birla Cancer Research Institute Satna,

Madhya Pradesh, India

E-mail: rcagrawa160@yahoo.com 